J. Urol. Urogynäkol. AT 2020 • 27:66-69 https://doi.org/10.1007/s41972-020-00094-7 Online publiziert: 28. Februar 2020 (c) Der/die Autor(en) 2020
Nathalie Garstka · Shahrokh François Shariat

Universitätsklinik für Urologie, Medizinische Universität Wien, Wien, Österreich

\title{
Metastasenchirurgie beim Harnblasenkarzinom: Sinn oder Unsinn
}

Das Blasenkarzinom ist bei der Erstdiagnose in $20 \%$ der Fälle muskelinvasiv. Während das krankheitsspezifische 5-Jahres-Überleben bei nichtmuskelinvasiven Tumoren nach radikaler Zystektomie über $90 \%$ beträgt, wird es im Gegensatz dazu bei lokal fortgeschrittenen Tumorstadien mit Lymphknotenmetastasen mit $<20 \%$ angegeben [1]. Trotz kurativ intendierter Therapie entwickeln etwa $40 \%$ der Patienten mit einem Urothelkarzinom der Harnblase ein Rezidiv mit eventuellen Metastasen. Die oftmals daraufhin eingesetzte cisplatinbasierte Standardchemotherapie erzielt Tumorremissionsraten in bis zu $70 \%$ [2]. Nichtsdestotrotz erreichen lediglich $15 \%$ ein medianes 5-JahresÜberleben; eine nachhaltige Remission kann in diesem Setting nur sehr selten erreicht werden, dies hauptsächlich bei Lymphknotenmetastasen [3]. Zudem ist etwa die Hälfte der mUC-Patienten Cisplatin-ungeeignet, meistens aufgrund einer Niereninsuffizienz. Chemotherapeutische Alternative ist ein carboplatinbasiertes Regime, dessen Effektivität dem Cisplatinschema allerdings unterlegen ist. Durch die Einführung von Checkpointinhibitoren konnte für Patienten mit mUC, erstmals seit vielen Jahrzehnten, eine relevante Verbesserung erzielt werden - sowohl in der Erstlinie für cisplatinungeeignete Patienten oder jene, die unter platinhaltiger Erstlinienchemotherapie einen Progress erleiden. Ebenso scheint eine Kombination aus Immunund Chemotherapie vielversprechend zu sein. Auf dem „European Congress of Medical Oncology“ (ESMO) 2019 wurde die Zwischenanalyse der Phase3-Studie IMvigor130 vorgestellt. Hierbei zeigte sich für die Kombination Atezolizumab + Gemcitabin/Cisplatin eine signifikante Verlängerung des progressionsfreien Überlebens im Vergleich zur alleinigen Chemotherapie (8,2 vs. 6,3 Monate, $p<0,007)$. Die statistische Signifikanz und der Impact auf das Überleben müssen im Follow-up noch evaluiert werden [4].

Neben der Weiterentwicklung der medikamentösen Therapie steht die chirurgische Intervention in gut selektierten Patienten mit mUC. Eine Metastasenresektion, als etablierter Bestandteil eines multimodalen Therapiekonzepts mit Intention der Lebensverlängerung und/ oder Erhöhung der Lebensqualität gehört bei anderen soliden bösartigen Tumoren, wie dem Hoden- oder Kolonkarzinom, zum Standard [5]. Metastasenresektionen beim Urothelkarzinom wurden erstmals in den frühen 1980er Jahren beschrieben. Cowles et al. berichteten damals ein Langzeitüberleben von 5 Jahren bei 6 Patienten, bei denen eine Lungenteilresektion aufgrund solitärer pulmonaler Filiae durchgeführt wurde [6]. Die in Folge dazu veröffentlichten Studien zur Evaluierung einer Metastasenchirurgie sind fast ausschließlich retrospektiv und beinhalten wenige Patientenzahlen [7].

Eine im Jahre 2018 veröffentliche Studie von Faltas et al. analysierte das Outcome von 497 älteren mUC-Patienten über 65 Jahre nach Metastasektomien aller Lokalisationen [8]. Hierbei wurden Patienten mit unterschiedlichen Absiedelungsorten inkludiert, welche präoperativ nicht oder chemotherapeutisch vorbehandelt wurden. Im Median überlebten die Patienten 19 Monate. In dieser se- lektiven Population überlebte nach 3 Jahren etwa 1/3 der Erkrankten. Der Krankenhausaufenthalt belief sich im Median auf7 Tage. Die beobachtete postoperative 30 -Tages-Mortalität lag bei $10 \%$ und ist somit höher als die postoperative Mortalität, die mit einer primären radikalen Zystektomie bei Patienten über 65 Jahren assoziiert ist [8]. Daraus lässt sich ableiten, dass eine adäquate Patientenselektion für eine Metastasenchirurgie notwendig ist.

\section{Klinisch positive Lymphknoten- metastasen}

Grossman et al. konnten mit der Phase3-Studie SWOG S8710 zeigen, dass Patienten mit T2-T4N0M0-Blasenkarzinom ein um $25 \%$ geringeres Sterberisiko aufweisen, wenn sie vor geplanter radikaler Zystektomie eine neoadjuvante Chemotherapie (NAC) erhielten [9]. Die meisten randomisierten NAC-Studien, welche einen Überlebensbenefit nachweisen, inkludierten sehr selten Patienten mit klinisch positiven Lymphknoten. Einige Studien befassten sich jedoch mit dem Outcome in einem cN+-Setting. Hierbei wurden innerhalb eines multimodalen Konzepts eine präoperative Chemotherapie und anschließend eine radikale Zystektomie mit Lymphadenektomie durchgeführt. Zargar-Shoshtari et al. untersuchten hierzu 304 Patienten mit klinischem Nachweis eines Lymphknotenbefalls (cN1-cN3); $42 \%$ erhielten eine induktive Chemotherapie mit Methotrexat/Vinblastin/Doxorubicin/Cisplatin, $43 \%$ mit Gemcitabin/Cisplatin und 15\% mit einem anderen Schema. Die pN0Rate war $48 \%$ (cN1: $56 \%$, cN2: $39 \%$, 
cN3: $39 \%, p=0,03)$. Ein komplettes Ansprechen zeigten $14,5 \%$. Die mittlere Gesamtüberlebenszeit für die Kohorte betrug 22 Monate. Eine Lymphknotenentfernung von >15 Lymphknoten, ein postoperativer $\mathrm{pN} 0$ - sowie $\mathrm{pR} 0$-Status und eine cisplatinbasierte Chemotherapie waren mit einem verlängerten Überleben assoziiert. Interessanterweise gab es keinen statistisch signifikanten Unterschied im Überleben zwischen den Patienten mit regionalem Lymphknotenbefall und den nach TNM-Klassifikation als fernmetastiert geltenden Patienten mit cN2-cN3-Status [10, 11]. Galsky et al. untersuchten 2016 Daten von 1739 mUC-Patienten aus der „National Cancer Data Base“ zwischen 2003 und 2012. In diesem Kollektiv befanden sich Patienten mit fortgeschrittenem Blasenkarzinom cT1-4cN1-cN3cM0 (cN1: $48 \%$; cN2. $45 \%$; cN3: $7 \%$ ), die entweder eine Chemotherapie und/oder radikale Zystektomie erhielten. Von den Zystektomierten erhielten 363 eine präoperative und 328 eine adjuvante Chemotherapie. Das 5-Jahres-Überleben für Chemotherapie allein, radikale Zystektomie allein, präoperative Chemotherapie gefolgt von radikaler Zystektomie und radikaler Zystektomie gefolgt von adjuvanter Chemotherapie betrug jeweils $14 \%, 19 \%, 31 \%$ und $26 \%$. Im Vergleich zur alleinigen radikalen Zystektomie war die induktive Chemotherapie mit einer signifikanten Verbesserung des Überlebens verbunden [12].

Die pathologische Tumorausdehnung und der Lymphknotenstatus sind die wichtigsten Prognosefaktoren, sowohl für das krankheitsspezifische als auch das rezidivfreie Überleben [13, 14]. Insbesondere das Vorhandensein vitaler Lymphknotenmetastasen nach induktiver Chemotherapie wirkt sich negativ auf das krankheitsspezifische Überleben aus [7]. Dass der pN-Status nach Chemotherapie ein stärkerer Prädiktor für das Überleben als die lokale Tumorausdehnung ist, konnten Nieuwenhuijzen et al. zeigen. Insgesamt 52 Blasenkarzinompatienten mit histologisch positiven Lymphknoten (durch Lymphknotendissektion oder Aspirationszytologie nachgewiesen) wurden chemotherapeutisch behandelt und bei partieller oder

J. Urol. Urogynäkol. AT 2020 · 27:66-69 https://doi.org/10.1007/s41972-020-00094-7

(c) Der/die Autor(en) 2020

\section{N. Garstka $\cdot$ S. Shariat}

\section{Metastasenchirurgie beim Harnblasenkarzinom: Sinn oder Unsinn}

\section{Zusammenfassung}

Das Outcome eines lymphknotenpositiven oder fernmetastasierten Urothelkarzinoms (mUC) der Harnblase ist schlecht. Eine cisplatinbasierte Chemotherapie wie Gemcitabin/Cisplatin oder akzeleriertes MVAC (Methotrexat, Vinblastin, Adriamycin und Cisplatin) bilden den Standard in der systemischen Erstlinientherapie des mUC. Alternativ kann seit 2016 in diesem Setting eine Immuntherapie angeboten werden. Somit stehen z. B. in der Erstlinie cisplatinungeeigneten Patienten die Checkpointinhibitoren Atezolizumab oder Pembrolizumab zur Verfügung, sofern sie einen positiven PD-L1-
Status aufweisen. Eine Metastasen gezielte operative Therapie wird bereits erfolgreich bei verschiedenen urologischen Tumoren, wie dem Hoden- oder dem Nierenzellkarzinom, eingesetzt, um das Überleben zu verlängern und/oder die Lebensqualität zu verbessern. In diesem Artikel soll der Stellenwert der Metastasenchirurgie beim mUC der Harnblase evaluiert werden.

\section{Schlüsselwörter}

Fortgeschrittene Krebserkrankung · Blasenkarzinom - Metastasektomie . Metastasiertes Urothelkarzinom · Chirurgie

\section{Surgery for metastasis in bladder cancer: does it make sense or not?}

\section{Abstract}

The outcome of lymph node-positive or distant metastatic urothelial carcinoma (mUC) of the bladder is poor. Cisplatin-based chemotherapy such as gemcitabine/cisplatin or accelerated MVAC (methotrexate, vinblastine, adriamycin and cisplatin) represent the standard systemic first-line therapy of mUC. Alternatively, immunotherapy has been available to metastatic patients since 2016. The checkpoint inhibitors atezolizumab or pembrolizumab are available for the treatment of cisplatin-unsuitable patients with a positive PD-L1 status. Cytoreductive or surgical therapy of metastasis is already being successfully used in various urologic tumors such as testicular or renal cell carcinoma with the intention of potentially prolonging survival. The aim of this article is to evaluate the importance of metastatic surgery and improving quality of life in patients with mUC of the urinary bladder.

\section{Keywords}

Advanced malignancy · Bladder carcinoma . Metastasectomy · Metastatic urothelial carcinoma $\cdot$ Surgery vollständiger Remission anschließend einer radikalen Zystektomie unterzogen. Komplette Remission, partielle Remission und stabile/progressive Erkrankung wurden in $29 \%, 57 \%$ und $14 \%$ erreicht und führten zu einem 5-Jahres-Überleben von jeweils $42 \%, 19 \%$ bzw. $0 \%$. Kein Patient mit einem positiven $\mathrm{pN}$ Status überlebte mehr als 2 Jahre nach der Operation, was die Bedeutung der Lymphknotenreaktion auf die Chemotherapie unterstreicht [15].

Herr et al. publizierten eine Studie mit 207 Patienten mit einem lokal fortgeschrittenen Urothelkarzinom oder primären cN1-Status. 39\% der Patienten erhielten eine Metastasenresektion nach Beendigung der Chemotherapie. Bei den anderen $61 \%$ waren entweder bereits unter der Chemotherapie eine Progredienz nachweisbar oder eine Operation aufgrund eines schlechten Performancestatus nicht möglich. Von den Operierten erreichten 44 eine partielle und 24 eine komplette Remission, während 12 nicht auf die Chemotherapie ansprachen. Die chirurgische Metastasenentfernung ergab in $30 \%$ der Fälle eine Nekrose. In diesem Fall lag das 5-Jahres-Überleben bei $58 \%$. Bei 49 (61\%) Patienten war noch aktives Karzinomgewebe in den vollständig resezierten Residuen vorhanden, von denen $41 \%$ überlebten. Von den 127 Patienten ohne Metastasenresektion überlebte nur 1 [16]. 


\section{Fernmetastasen}

Die häufigsten Metastasenlokalisationen beim Blasenkarzinom sind Lymphknoten, Lunge, Leber und Knochen [17]. Im Falle einer Streuung treten diese in $90 \%$ der Fälle bereits innerhalb der ersten 3 Jahre nach einer radikalen Zystektomie auf. Nach Standardtherapie mit einer cisplatinhaltigen Chemotherapie überleben metastasierte Patienten im Median knapp 1-2 Jahre. Otto et al. evaluierten die Rolle einer Metastasektomie bei 70 Patienten, die unter MVAC-Chemotherapie eine Progression zeigten. Die meisten Patienten hatten multiple $\mathrm{Me}$ tastasen (76\%), bei $41 \%$ waren mehrere Organe beteiligt. Die mediane Überlebenszeit betrug 7 Monate und war somit kürzer als bei alleiniger Chemotherapie. Es ergab sich kein Überlebensvorteil für die Metastasektomie und kein Unterschied im Überleben zwischen verschiedenen Metastasenlokalisationen. Bezogen auf die Lebensqualität profitierten allerdings insbesondere Patienten mit symptomatischer Metastasierung von einer Operation, währenddessen zuvor asymptomatische Patienten über eine Reduktion des Wohlbefindens klagten [18]. Siefker-Radtke et al. analysierten das Outcome von 31 Patienten, welche bei einem mUC eine Metastasenresektion erhielten. Davon erfolgte die Metastasenchirurgie bei 22 nach Chemotherapie und bei 9 primär. Absiedelungen fanden sich in der Lunge $(n=24)$, in überregionalen Lymphknoten $(n=4)$, im Gehirn $(n=2)$ und subkutan $(n=1)$. Eine vollständige Entfernung gelang bei 30 Patienten. Im Median überlebten die Patienten 23 Monate. Das 5-JahresÜberleben lag bei $33 \%$. Bis zu einer nachweisbaren Krankheitsprogredienz vergingen im Median allerdings nur 7 Monate. In dieser Studie machte es keinen Überlebensunterschied, ob die Filiae nach einer Chemotherapie oder direkt reseziert wurden. Auch spielte es keine Rolle, ob einzelne oder mehrere Metastasen vorhanden waren [19].

Abe et al. untersuchten das Outcome eines ebenfalls heterogen metastasierten Patientenkollektivs, bestehend aus 15 primär und $33 \mathrm{im}$ Verlauf metastasierten Patienten. Lediglich bei 2 Patienten ent- hielten entnommene suspekte Lymphknoten und ein Lokalrezidiv vitales Tumorgewebe. Patienten mit Metastasenresektion überlebten 42 Monate, nichtoperierte lediglich 10 Monate. In der multivariaten Analyse waren $>5$ verabreichte Zyklen Chemotherapie, eine operative Metastasenentfernung sowie die Abwesenheit eines Lokalrezidivs und von Leber- und Knochenfiliae positiv mit einem verlängerten Überleben assoziiert [20]. Einige Arbeitsgruppen untersuchten den Einfluss des Metastasenortes. Dabei zeigte sich ein günstigeres Outcome für Patienten, welche sich einer chirurgischen Sanierung von Lungenmetastasen unterzogen hatten. Kanzaki et al. berichteten von 18 Urothelkarzinompatienten, bei denen pulmonale Metastasen entfernt wurden. Nach 3 Jahren lebten noch 59,8\%, nach 5 Jahren 46,5\% der Erkrankten. Die 5-Jahres-Überlebensrate von Patienten mit solitären Metastasen betrug 85,7\%, während die von Patienten mit Mehrfachmetastasen $20 \%$ betrug [21]. Matsuguma et al. zeigten bei 32 pulmonal Metastasektomierten eine 5-Jahres-Überlebensrate von $50 \% .2$ weitere Studien betonten den prognostisch günstigeren Impact einer alleinigen pulmonalen Metastasierung, wobei sich eine Metastasengröße von $<3 \mathrm{~cm}$ als besonders günstiger Faktor erwies und mit längerem Überleben assoziiert war [14, 22].

\section{Fazit für die Praxis}

- Die chirurgische Metastasenresektion beim metastasierten Urothelkarzinom (mUC) der Harnblase kann als Teil eines individuellen Therapiekonzeptes eingesetzt werden.

- Die Datenlage zur Metastasenchirurgie ist schwach, und die Evidenzlage gering.

- Für eine sehr selektierte Patientengruppe zeichnet sich ein Benefit im Falle einer Metastasektomie ab. Patienten mit Lymphknotenmetastasen, welche auf eine induktive Chemotherapie ansprechen, profitieren wahrscheinlich von einer anschlieBenden Lymphknotendissektion.

- Zumindest im Falle von symptomatischen Metastasen besteht die Möglichkeit, durch die Entfernung von schmerzenden oder einschränkenden Metastasen die Lebensqualität zu verbessern.

- Mit Fortschreiten medizinischer Entwicklungen, u.a. bei den bildgebenden Verfahren (z.B. FDG-PET), wird es ein neues Interesse an der Metastasektomie geben. Hierbei wird es eine Verschiebung zu aggressiveren lokalen Therapien geben, sei es chirurgisch oder durch stereotaktische Radiotherapie.

\section{Korrespondenzadresse

Nathalie Garstka, BSc
Universitätsklinik für Urologie,
Medizinische Universität
Wien
Währinger Gürtel 18-20,
1090 Wien, Österreich
nathalie.garstka@
meduniwien.ac.at

Funding. Open access funding provided by Medical University of Vienna.

\section{Einhaltung ethischer Richtlinien}

Interessenkonflikt. N. Garstka und S. Shariat geben an, dass kein Interessenkonflikt besteht.

Für diesen Beitrag wurden von den Autoren keine Studien an Menschen oder Tieren durchgeführt. Für die aufgeführten Studien gelten die jeweils dort angegebenen ethischen Richtlinien.

Open Access Dieser Artikel wird unter der Creative Commons Namensnennung 4.0 International Lizenz veröffentlicht, welche die Nutzung, Vervielfältigung, Bearbeitung, Verbreitung und Wiedergabe in jeglichem Medium und Format erlaubt, sofern Sie den/die ursprünglichen Autor(en) und die Quelle ordnungsgemäß nennen, einen Link zur Creative Commons Lizenz beifügen und angeben, ob Änderungen vorgenommen wurden

Die in diesem Artikel enthaltenen Bilder und sonstiges Drittmaterial unterliegen ebenfalls der genannten Creative Commons Lizenz, sofern sich aus der Abbildungslegende nichts anderes ergibt. Sofern das betreffende Material nicht unter der genannten Creative Commons Lizenz steht und die betreffende Handlung nicht nach gesetzlichen Vorschriften erlaubt ist, ist für die oben aufgeführten Weiterverwendungen des Materials die Einwilligung des jeweiligen Rechteinhabers einzuholen.

Weitere Details zur Lizenz entnehmen Sie bitte der Lizenzinformation auf http://creativecommons.org/ licenses/by/4.0/deed.de. 


\section{Literatur}

1. Shariat SF, Karakiewicz PI, Palapattu GS, Lotan Y, Rogers CG, Amiel GE et al (2006) Outcomes of radical cystectomy for transitional cell carcinoma of the bladder: a contemporary series from the Bladder Cancer Research Consortium. J Urol 176(6 Pt 1):2414-2422. https://doi.org/10.1016/j.juro. 2006.08.004 (discussion 2422)

2. Sternberg CN, Yagoda A, Scher HI, Watson RC, Geller N, Herr HW et al (1989) Methotrexate, vinblastine, doxorubicin, and cisplatin for advanced transitional cell carcinoma of the urothelium. Efficacy and patterns of response and relapse. Cancer 64(12):2448-2458

3. Von der Maase H, Sengelov L, Roberts JT, Ricci S, Dogliotti L, Oliver T et al (2005) Longterm survival results of a randomized trial comparing gemcitabine plus cisplatin, with methotrexate, vinblastine, doxorubicin, plus cisplatin in patients with bladder cancer. J Clin Oncol 23(21):4602-4608.https://doi.org/10.1200/ JCO.2005.07.757

4. Grande E, Galsky M, Arranz Arija JA www.esmo. org/Oncology-News/Adding-Atezolizumab-toFront-Line-Platinum-Based-ChemotherapyImproves-Progression-Free-Survival-in-mUC. Zugegriffen: 31.01.2020

5. Frankel TL, D'Angelica MI (2014) Hepatic resection for colorectal metastases. J Surg Oncol 109(1):2-7. https://doi.org/10.1002/jso.23371

6. Cowles RS, Johnson DE, McMurtrey MJ (1982) Long-term results following thoracotomy for metastatic bladder cancer. Urology 20(4):390-392. https://doi.org/10.1016/0090-4295(82)90462-9

7. Abufaraj M, Dalbagni G, Daneshmand S, Horenblas S, Kamat AM, Kanzaki R et al (2018) The role of surgery in metastatic bladder cancer: a systematic review. Eur Urol 73(4):543-557. https://doi.org/10. 1016/j.eururo.2017.09.030

8. Faltas BM, Gennarelli RL, Elkin E, Nguyen DP, Hu J, Tagawa ST (2018) Metastasectomy in older adults with urothelial carcinoma: populationbased analysis of use and outcomes. Urol Oncol36(1):9.e11-9.e17.https://doi.org/10.1016/ j.urolonc.2017.09.009

9. Grossman HB, Natale RB, Tangen CM, Speights VO, Vogelzang NJ, Trump DL et al (2003) Neoadjuvant chemotherapy plus cystectomy compared with cystectomy alone for locally advanced bladder cancer. N Engl J Med 349(9):859-866. https://doi. org/10.1056/NEJMoa022148

10. Zargar-Shoshtari K, Zargar H, Lotan $Y$, Shah JB, van Rhijn BW, Daneshmand S et al (2016) A multiinstitutional analysis of outcomes of patients with clinically node positive urothelial bladder cancer treated with induction chemotherapy and radical Cystectomy. J Urol 195(1):53-59. https://doi.org/ 10.1016/j.juro.2015.07.085

11. Paner GP, Stadler WM, Hansel DE, Montironi $R$, Lin DW, Amin MB (2018) Updates in the eighth edition of the tumor-node-metastasis staging classification for urologic cancers. Eur Urol 73(4):560-569. https://doi.org/10.1016/j.eururo. 2017.12.018

12. Galsky MD, Stensland K, Sfakianos JP, Mehrazin R, Diefenbach M, Mohamed N et al (2016) Comparative effectiveness of treatment strategies for bladder cancer with clinical evidence of regional lymph node involvement. J Clin Oncol 34(22):2627-2635 https://doi.org/10.1200/JCO.2016.67.5033

13. Stein JP, Lieskovsky G, Cote R, Groshen S, Feng AC, Boyd $S$ et al (2001) Radical cystectomy in the treatment of invasive bladder cancer: long- term results in 1,054 patients. J Clin Oncol 19(3):666-675. https://doi.org/10.1200/JCO.2001. 19.3.666

14. Matsuguma $H$, Yoshino I, Ito $H$, Goya T, Matsui $Y$, Nakajima J et al (2011) Is there a role for pulmonary metastasectomy with a curative intent in patients with metastatic urinary transitional cell carcinoma? Ann Thorac Surg 92(2):449-453. https://doi.org/10.1016/j.athoracsur.2011.03.097

15. Nieuwenhuijzen JA, Bex A, Meinhardt W, Kerst JM, Schornagel JH, van Tinteren $\mathrm{H}$ et al (2005) Neoadjuvant methotrexate, vinblastine, doxorubicin and cisplatin for histologically proven lymph node positive bladder cancer. J Urol 174(1):80-85. https:// doi.org/10.1097/01.ju.0000162018.40891.ba

16. Herr HW, Donat SM, Bajorin DF (2001) Post-chemotherapy surgery in patients with unresectable or regionally metastatic bladder cancer. J Urol 165(3):811-814

17. Bochner BH, Montie JE, Lee CT (2003) Followup strategies and management of recurrence in urologic oncology bladder cancer. Urol Clin North Am 30(4):777-789. https://doi.org/10. 1016/s0094-0143(03)00061-2

18. Otto T, Krege S, Suhr J, Rübben H (2001) Impact of surgical resection of bladder cancer metastases refractory to systemic therapy on performance score: a phase II trial. Urology 57(1):55-59. https:// doi.org/10.1016/s0090-4295(00)00867-0

19. Siefker-Radtke AO, Walsh GL, Pisters LL, Shen Y, Swanson DA, Logothetis CJ et al (2004) Is there a role for surgery in the management of metastatic urothelial cancer? The M. D. Anderson experience. J Urol 171(1):145-148. https://doi.org/10.1097/ 01.ju.0000099823.60465.e6

20. Abe T, Shinohara N, Harabayashi T, Sazawa A, Maruyama S, Suzuki S et al (2007) Impact of multimodal treatment on survival in patients with metastatic urothelial cancer. Eur Urol 52(4):1106-1113. https://doi.org/10.1016/j.eururo.2007.02.052

21. Kanzaki R, Higashiyama M, Fujiwara A, Tokunaga T, Maeda J, Okami J et al (2010) Outcome of surgical resection of pulmonary metastasis from urinary tract transitional cell carcinoma. Interact CardioVasc Thorac Surg 11(1):60-64. https://doi. org/10.1510/icvts.2010.236687

22. Luzzi L, Marulli G, Solli P, Cardillo G, Ghisalberti M, Mammana $\mathrm{M}$ et al (2017) Long-term results and prognostic factors of pulmonary metastasectomy in patients with metastatic transitional cell carcinoma. Thorac Cardiovasc Surg 65(7):567-571. https://doi.org/10.1055/s-0036-1583271

Hinweis des Verlags. Der Verlag bleibt in Hinblick auf geografische Zuordnungen und Gebietsbezeichnungen in veröffentlichten Karten und Institutsadressen neutral.

\section{Daniela Pucher \\ Zur Sache, Experten!}

Sachbuch schreiben und vermarkten. Eine 10-Schritte-Anleitung

Wien: Springer 2019, 1. Aufl., 228 S. 47 Abb., (ISBN: 978-3-662-59224-3), 20,55 EUR

\section{Zur Sache, Experten!}

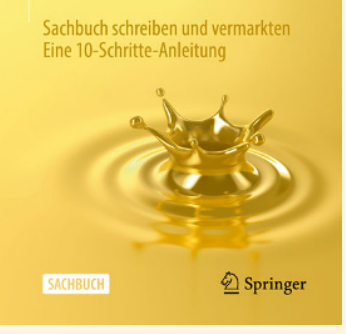

Was unterscheidet erfolgreiche Speaker, Berater, Coachs, Therapeuten oder andere Spezialisten von weniger erfolgreichen? Sie haben ein Sach-oder Fachbuch geschrieben. Ein Buch ist die wohl edelste aller Visitenkarten für Experten und Wissende. Und heute, in Zeiten des Content-Marketing, lässt es sich auch noch gekonnt in die Kommunikations- und Marketingstrategie einfügen.

Damit das gelingt, braucht es zweierlei: Das Buch muss professionell geschrieben und publiziert werden und es muss die Expertise des Autors inhaltlich und persönlich repräsentieren, um als Marketinginstrument perfekt einsetzbar zu sein.

Um das zu bewerkstelligen, hilft dieses Buch. Mit vielen launigen Geschichten aus der langjährigen Erfahrung als Autorenberaterin und Ghostwriterin gibt Daniela Pucher Einblick hinter die Kulissen des Buchkonzipierens, Schreibens und Vermarktens. Daniela Puchner zeigt wie man Leser begeistert, Aufmerksamkeit bekommt und Kunden gewinnt. Mit Hilfe ihrer 10-Schritte-Anleitung haben Sie am Ende ein erfolgreiches Buch in Händen, das bestmöglichen Absatz verspricht. Für all jene gedacht, die ihren Bekanntheitsgrad erhöhen, neue Kunden gewinnen oder sich als Experte einen Namen machen möchten. 